\title{
Utilización del Diseño de Experimentos para la Optimización de Mezclas
}

\section{Use of Design of Experiments for the Optimization of Mixtures}

\section{Néstor 0. Ulibarrie}

CECOVI - Centro de Investigación y Desarrollo para la Construcción y la Vivienda. Universidad Tecnológica Nacional Facultad Regional Santa Fe.

nulibarr@frsf.utn.edu.ar

\section{Loreley B. Beltramini}

CECOVI - Centro de Investigación y Desarrollo para la Construcción y la Vivienda. Universidad Tecnológica Nacional Facultad Regional Santa Fe.

Ibeltramini@frsf.utn.edu.ar

\section{Natacha L. Bourquen}

CECOVI - Centro de Investigación y Desarrollo para la Construcción y la Vivienda. Universidad Tecnológica Nacional Facultad Regional Santa Fe.

natachabourquen@hotmail.com

\section{Victoria Defagot}

CECOVI - Centro de Investigación y Desarrollo para la Construcción y la Vivienda. Universidad Tecnológica Nacional Facultad Regional Santa Fe.

victoriadefagot@gmail.com

\section{Federico Andrés}

CECOVI - Centro de Investigación y Desarrollo para la Construcción y la Vivienda. Universidad Tecnológica Nacional Facultad Regional Santa Fe.

fede_andres03@hotmail.com 


\title{
Resumen
}

Se presenta una propuesta para convertir desechos de material celulósico en compuestos con un valor agregado que tengan un potencial uso en la industria de la construcción. El desarrollo se basa en la producción de paneles de revestimiento interior a partir de la mezcla de tres componentes: residuo de celulosa, cemento y agua.

Para generar la mezcla optimizada de estos componentes se optó por la utilización de un Diseño de Experimentos, que permite reducir la cantidad de muestras a fabricar tomando como punto de referencia una superficie de respuesta optimizada.

A partir del análisis de resultados se observa una relación directa entre la conductividad térmica y la densidad del material ya que, a medida que la densidad aumenta también lo hace el coeficiente de conductividad térmica. Se espera que los paneles formen parte de la arquitectura sustentable que promueve el uso responsable de la energía para el acondicionamiento de las viviendas.

Palabras claves: Paneles, celulosa, aislación térmica, diseño de experimentos.

\begin{abstract}
A proposal is presented to convert wastes of cellulosic material into compounds with value added that have a potential use in the construction industry. The development is based on the production of interior lining panels from the mixture of three components: cellulose residue, cement and water.

In order to generate the optimized mixture of these three components, an experiment design was used to reduce the number of samples to be produced, taking as a reference point an optimized response surface.

From the analysis of results, a direct relationship between the thermal conductivity and the density of the material is observed since, as the density increases, so does its coefficient of conductivity. It is expected that the panels form part of the sustainable architecture that promotes the responsible use of energy for the conditioning of homes.
\end{abstract}

Key words: panels, cellulose, thermal insulation, design of experiments. 


\section{INTRODUCCIÓN}

La industria de la construcción posee una vasta experiencia en utilizar residuos dentro de las formulaciones de nuevos materiales. Ésta re-valorización puede producirse en grandes cantidades ya que esta industria es una excelente receptora de ciertos desechos o subproductos (Zanuttini M.A., 2012. Polo M., 2015). La posibilidad de reutilizar grandes cantidades de descartes la hace una alternativa altamente atractiva.

Este trabajo responde a la necesidad de preservar el medio ambiente, con el fin de lograr una disminución del volumen de desechos sólidos urbanos y una reducción de su incineración dentro de los rellenos sanitarios (acción que se realiza para mitigar el constante aumento de volumen de residuos) (Zanuttini M.A., 2012).

Se pretende entonces, evaluar la viabilidad de la producción de paneles atérmicos para ser utilizados en la construcción como material de revestimiento interior, generados a partir de un residuo industrial de una empresa cervecera de la Ciudad de Santa Fe (Figura 1).

El desecho que se utiliza está formado por etiquetas de los envases que retornan a la cervecería, compuestas principalmente por material celulósico, restos de soda cáustica, tintas contaminantes y aditivos incorporados que son necesarias para un buen desempeño de las mismas (Polo M., 2015).

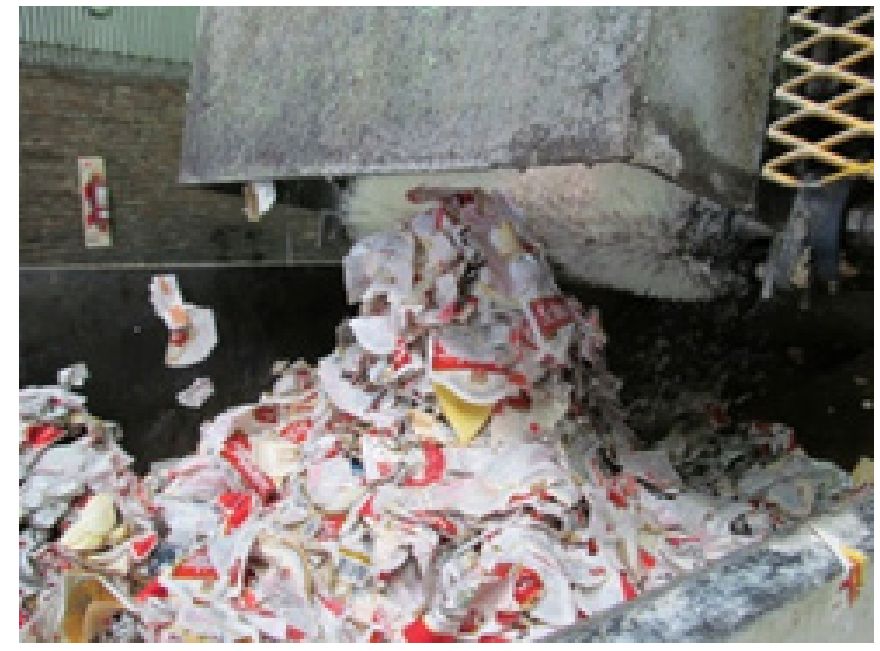

Figura 1. Residuo utilizado.

El proceso del reciclaje de dicho material para convertirlo nuevamente en papeles es limitado, debido a la mezcla particular de diferentes tipos de fibras que producen una pérdida de las propiedades específicas que podrían obtenerse de pulpas vírgenes, además de que dicho proceso implica el destintado de las etiquetas, lo cual genera residuos potencialmente contaminantes (Zanuttini M.A., 2012). Esto motiva a trabajar con dicho material para otras utilidades que no sea el papel reciclado, empleándolo como materia prima de materiales de construcción (Polo M., 2015. Bentchikou M. et al., 2012).

La utilización de fibras celulósicas en la fabricación de compuestos de cemento se encuentra escasamente reflejado en bibliografía. Bentchikou et al. estudiaron el efecto de 
utilizar fibras celulósicas como reemplazo del cemento en las propiedades de un compuesto ligero de cemento. Las fibras fueron obtenidas a partir de papeles y cajas de embalaje, luego de un blanqueado con agua y solución de hipoclorito de sodio. Se observó una reducción general en la resistencia a la compresión del concreto con el aumento del contenido de fibras. Sin embargo, las propiedades de aislamiento térmico se incrementaron. El material mostró una distribución homogénea de fibras en la matriz para relaciones de agua/cemento adecuadas para diferentes contenidos de fibras. Además, con el aumento del porcentaje de fibras la densidad aparente disminuyó, lo que permite producir materiales de construcción más livianos (Neithalath, N.et al., 2004).

En la confección de materiales aislantes, se han publicado trabajos a partir de pasta de papel reciclado como agregados para morteros y hormigones (Muñoz A. et al., 2006). Además, se realizaron investigaciones acerca de la conductividad térmica de materiales de desecho: cenizas de carbón y material cementante conteniendo celulosa de papel reciclado. Los ensayos se realizaron a temperaturas de uso industrial, con el fin de ser utilizados como aislantes. Se observó que ambos materiales exhibían buenas propiedades aislantes, y que su conductividad térmica disminuía con el aumento de la temperatura, pudiéndose emplear en equipos cuya operación normal se encuentra cerca de los $300^{\circ} \mathrm{C}$.

En estudios realizados previamente sobre la combinación de los componentes: cemento, celulosa y agua, se ha determinado que no es posible obtener, a partir de la mezcla de éstos, un material resistente y de baja densidad (Andrés, F.et al., 2013). Esto condujo a descartar la búsqueda de un material resistente y plantear un trabajo para el desarrollo de un elemento constructivo aislante térmico (Neithalath, N.et al., 2004. Muñoz, A. et al., 2006), evaluando la optimización de la mezcla. Para ello, se decidió utilizar una herramienta sistemática, como lo es el Diseño de Experimentos que permite hacer pruebas de manera más eficaz, ya que la potencialidad del mismo consiste en determinar qué pruebas se deben realizar y de qué manera para obtener datos que, al ser analizados estadísticamente, proporcionen evidencias objetivas que permitan responder los interrogantes planteados (Gutiérrez Pulido, H. et al., 2008. Menéndez, G. et al., 2008).

El Diseño de Experimentos se puede definir como la aplicación del método científico para la generación de conocimiento acerca de un sistema o proceso, por medio de pruebas planificadas adecuadamente, con el fin de obtener resultados con la máxima información y el menor trabajo y tiempo posible (Gutiérrez Pulido, H. et al., 2008).

\section{DESARROLLO}

El punto de partida fue generar un material de mediana densidad y baja conductividad térmica para la optimización de la mezcla de los materiales antes mencionados.

Para lograr este objetivo, se utilizó el Diseño de Experimentos puesto que es una herramienta que asume que los valores de las características medidas o resultados de los ensayos, dependen sólo de las proporciones relativas de los componentes de la mezcla, y en este caso, de las proporciones relativas de los tres componentes.

Debido a que hay infinidad de composiciones que se pueden elegir para formar parte del plan de experimentos, los diseños de mezcla parten de la base de una región experimental o de trabajo, acotada por restricciones impuestas (Polo, M., 2015).

Para el caso en estudio se tienen mezclas de tres componentes, los cuales forman una región experimental con forma de triángulo equilátero, donde en cada vértice se posiciona el compuesto puro, y entre cada vértice y la mitad de la base contraria (no adyacente) se 
considera un rango de fracción másica de 0 a 1 (Gutiérrez Pulido, H. et al., 2008. Menéndez, G. et al., 2008. Eriksson, L. et al., 1998. Jiao, D. et al., 2018).

El programa utilizado para la determinación del orden y la cantidad de experimentos fue MINITAB (Polo, M., 2015). El mismo requiere como datos un rango de valores de coeficientes de conductividad térmica y densidades que se desean obtener, y un rango de la cantidad de cada componente (Menéndez, G. et al., 2008. Eriksson, L. et al., 1998. Jiao, D. et al., 2018). El modelo está acotado en las siguientes condiciones para cada una de las variables como se muestra en la Tabla 1.

\begin{tabular}{|c|c|c|}
\hline Material & Máximo [g] & Mínimo [g] \\
\hline Cemento & 200 & 60 \\
\hline Papel & 30 & 10 \\
\hline Agua & 500 & 250 \\
\hline
\end{tabular}

Tabla 1: Cantidades para las cuales el modelo está acotado.

Esta restricción se realiza ya que, para algunas combinaciones de estos materiales, no es posible generar probetas aptas de ser ensayadas. A partir de estos valores se acota el modelo (Figura 2 y Figura 3), generando 13 experimentos para analizar las variables de respuesta antes mencionadas: densidad y conductividad térmica. Para cada uno de los experimentos se realizó una probeta sobre la cual se determinó la densidad y el valor de conductividad.

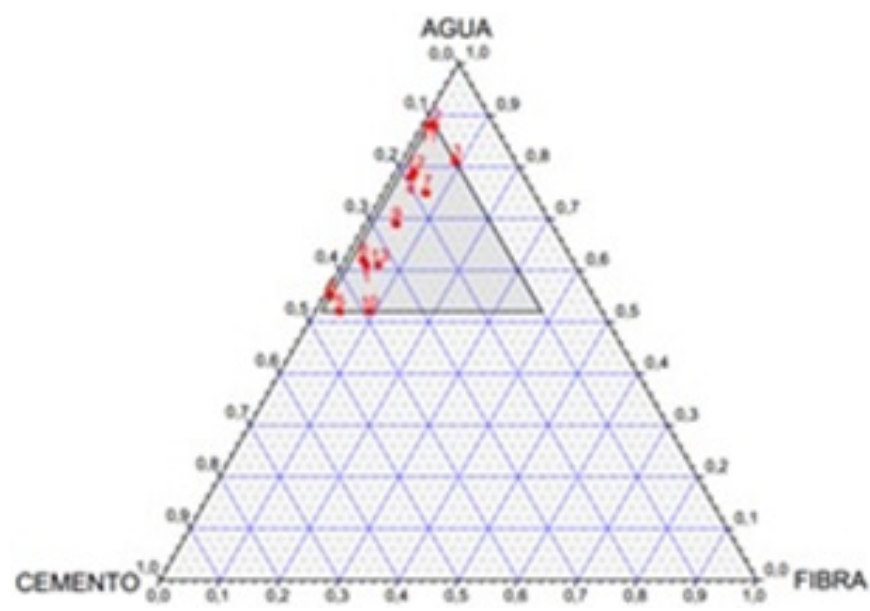

Figura 2. Gráfica de Diseño Simplex en proporciones. 


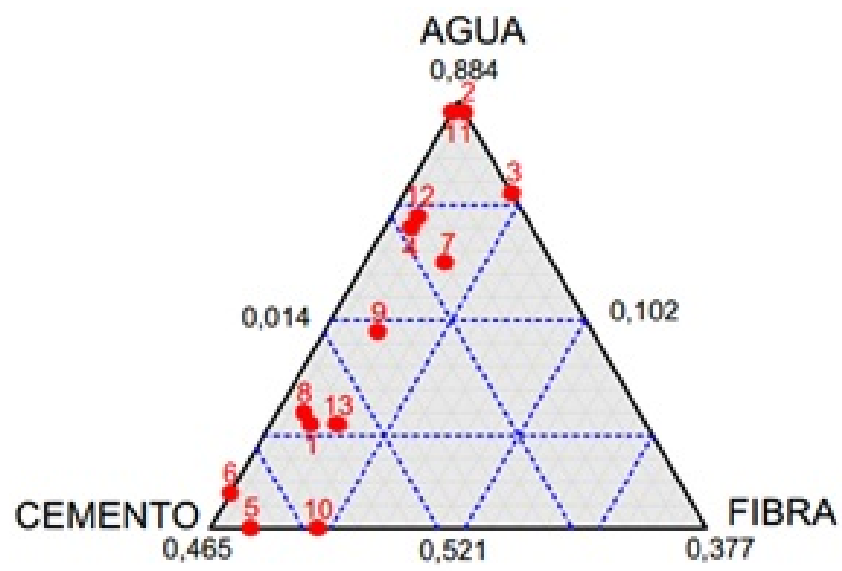

Figura 3. Fracción de la Gráfica de Diseño Simplex en proporciones correspondientes a las condiciones de estudio.

Para la fabricación de las probetas se utilizaron etiquetas procesadas con agua (Figura 4), y homogeneizadas con el cemento (Figura 5), cuya mezcla puede ser manipulada en estado fresco y luego, en estado endurecido, se obtiene un material de baja densidad.

La metodología de producción de las muestras es muy sencilla: se mezclaron manualmente los tres componentes de cada experimento hasta lograr la homogeneización. Luego, se vertió dicha mezcla sobre moldes preparados, de dimensiones apropiadas, para la posterior realización del ensayo de conductividad térmica. Al cabo de unos días se procedió a desmoldar las probetas y se continuó el proceso de secado en ambiente de laboratorio. A partir de allí, las probetas fueron secadas en estufa hasta que el peso de las mismas permaneció constante. Una vez alcanzada la constancia de peso, se determinó la densidad de las probetas.

Para la realización del ensayo de conductividad térmica fue necesario preparar las probetas de una manera especial debido a las características del equipo con que se determina este parámetro. El mismo se compone de dos placas metálicas, una de las cuales, la placa superior, proporciona calor y se transfiere, a través de la probeta a la placa inferior del aparato, el cual consta de instrumentos de medición de las temperaturas (Martina, P. et al., 2003).

Las probetas deben estar contenidas en un marco aislante para evitar la pérdida de calor lateral y así medir sólo la transferencia de calor transversal a las probetas (Kreith, F. et al., 2012) (Figura 6 y Figura 7).

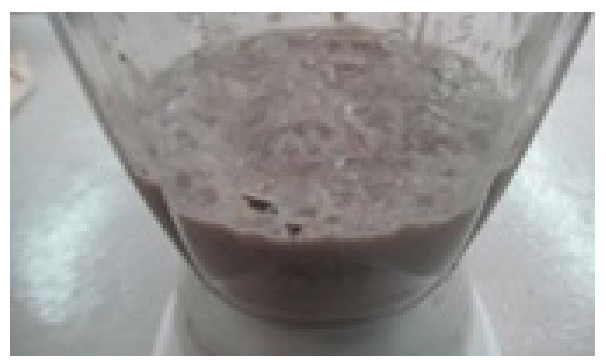




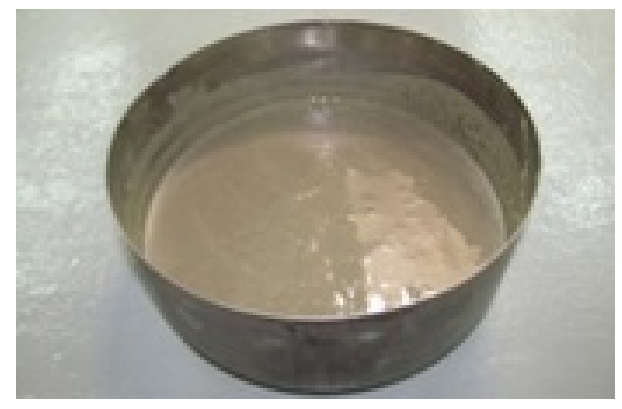

Figura 5.Homogenización de los componentes.

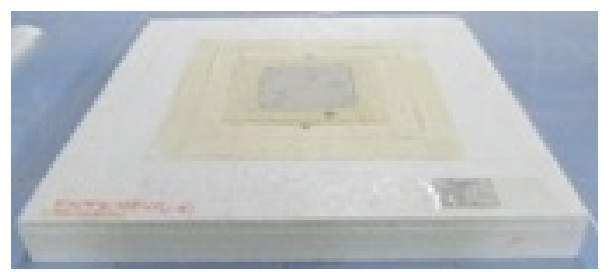

Figura 6. Probeta en condiciones de ser ensayada

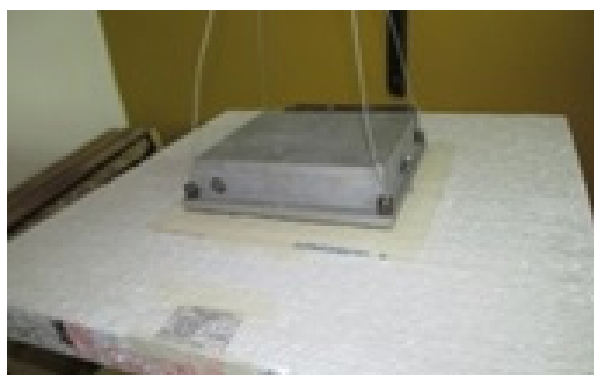

Figura 7. Ensayo a conductividad de una probeta

\section{RESULTADOS}

Durante el proceso de moldeo de las probetas de los 13 experimentos, se observaron distintos comportamientos de las mezclas efectuadas, presentando algunas de ellas una importante fluidez, dificultando el colado de probetas.

Sin embargo, todas las probetas tuvieron un comportamiento similar: se produjo una importante filtración de agua en la parte inferior del molde, motivo por el cual se originó un descenso de la altura de las mismas (modificando el rendimiento del material final), y como consecuencia se obtuvieron probetas de distintas densidades y espesores (Tabla 2).

Los resultados para cada uno de los parámetros evaluados se introdujeron en el programa MINITAB y como consecuencia se obtuvieron las superficies de respuesta del Diseño 
de Experimento planteado, ajustado a un modelo lineal (Gutiérrez Pulido, H. et al., 2008). Dichos resultados se muestran en las Figuras 9 y 10 para la respuesta densidad y conductividad térmica respectivamente.

\begin{tabular}{|c|c|c|}
\hline \multirow{2}{*}{$\begin{array}{c}\text { Experimento } \\
\mathrm{N}^{0}\end{array}$} & $\begin{array}{c}\text { Densidad } \\
\left(\mathrm{kg} / \mathrm{m}^{3}\right)\end{array}$ & $\begin{array}{c}\boldsymbol{\lambda} \text { promedio } \\
{\left[\mathrm{Kcal} / \mathrm{h}^{\circ} \mathrm{Cm}\right]}\end{array}$ \\
\hline 1 & 864 & 0,499 \\
\hline 2 & 597 & 0,249 \\
\hline 3 & 390 & 0,306 \\
\hline 4 & 603 & 0,318 \\
\hline 5 & 971 & 0,605 \\
\hline 6 & 1036 & 0,673 \\
\hline 7 & 508 & 0,424 \\
\hline 8 & 913 & 0,523 \\
\hline 9 & 658 & 0,350 \\
\hline 10 & 769 & 0,659 \\
\hline 11 & 431 & 0,220 \\
\hline 12 & 531 & 0,292 \\
\hline 13 & 697 & 0,472 \\
\hline
\end{tabular}

Tabla 2: Valores de densidad y conductividad de los experimentos.

Para interpretar la superficie de respuesta, se debe tener en cuenta que el efecto de un componente se define como el cambio en la respuesta a lo largo del eje que une este vértice con el centro del lado opuesto del simplex. A lo largo de este eje la proporción con la que participa este componente cambia de 1 a 0 , mientras que el resto de los componentes se mantienen en proporciones iguales, cuya suma cambia de 0 a 1 (Figura 8) (Gutiérrez Pulido, H. et al., 2008).

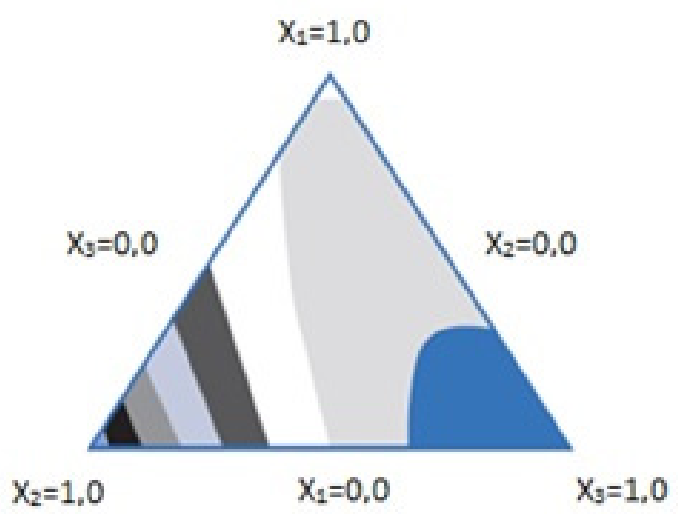

Figura 8. Superficie de respuesta modelo. 


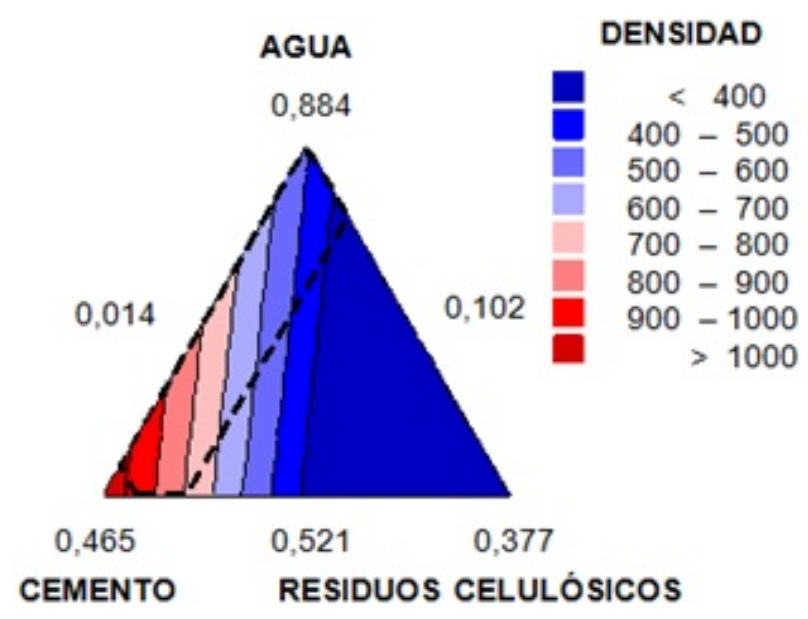

Figura 9. Superficie de respuesta de los valores de densidad de los experimentos.

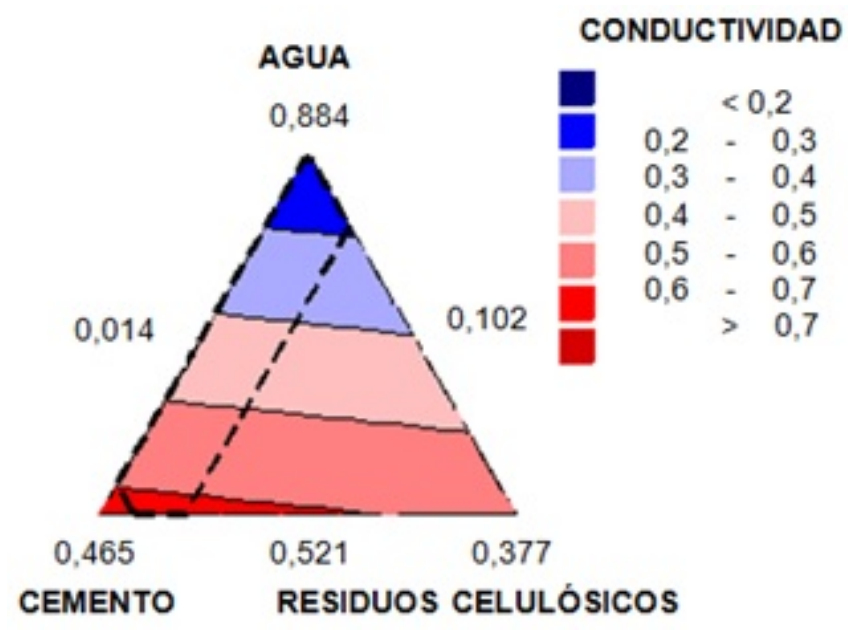

Figura 10. Superficie de respuesta de los valores de conductividad térmica de los experimentos.

\section{DISCUSIÓN}

Durante el moldeo de las probetas, se aprecia que al aumentar el contenido de fibra aumenta la absorción de agua de la mezcla, en coincidencia con lo encontrado por García-Ten et al., 2004.

Además, se observa una relación directa entre la densidad de los experimentos y sus coeficientes de conductividad térmica; aumentando el primero con el aumento de la densidad (Bentchikou M. et al., 2012). En esta dirección, se puede establecer también una correlación entre la densidad y el contenido de cemento, observándose un aumento de la densidad con el incremento de cemento, y como consecuencia, la probeta disminuye su capacidad aislante.

El valor de conductividad térmica de dicho experimento $\left(\lambda=0,306 \mathrm{Kcal} / \mathrm{h}^{\circ} \mathrm{Cm}\right)$ se lo compara con otros materiales destinados a proveer aislación, observándose que se encuentra en 
competencia con las placas de yeso que tienen un valor de $\lambda=0,37 \mathrm{Kcal} / \mathrm{h}^{\circ} \mathrm{Cm}$ y los revoques interiores de $0,93 \mathrm{Kcal} / \mathrm{h}^{\circ} \mathrm{Cm}$, valores aproximados en función del valor de densidad de cada material (Instituto Argentino de Normalización, 2002).

Asimismo, los resultados muestran que a medida que aumenta la cantidad de agua disminuye el valor del coeficiente de conductividad térmica; y a medida que aumenta la cantidad de fibra disminuye tanto la densidad como el coeficiente de conductividad térmica (Neithalath, N.et al., 2004).

\section{CONCLUSIONES}

Los mejores valores de conductividad térmica se presentaron en aquellas probetas que poseen mayor cantidad de agua y fibra y menor cantidad de cemento.

En relación al objetivo planteado en este trabajo se establece que el experimento $\mathrm{N}^{\mathrm{o}} 3$ es el que presenta mejores resultados en cuanto al valor del coeficiente de conductividad térmica, densidad, consistencia y trabajabilidad de la mezcla.

Se concluye que la destacada propiedad aislante, junto con su densidad favorable lo hacen un material apto y competitivo para su uso en la fabricación de paneles aislantes interiores.

Respecto a la utilización del Diseño de Experimentos, se facilitó la investigación tras la reducción en tiempo y experimentación. 


\section{REFERENCIAS}

Andrés, F. y Romano, M. (2013) "Hormigones Livianos: una alternativa para el reciclado de pasta celulósica”,Jornada de Investigadores Tecnológicos, JIT 2013, Universidad Tecnológica Nacional Facultad Regional Rafaela, Argentina, 8 de noviembre.

Bentchikou, M., Guidoum, A., Scrivener, K., Silhadi, K., y Hanini, S. (2012). Effect of recycled cellulose fibres on the properties of lightweight cement composite matrix. Construction and Building Materials, 34, 451-456.

Eriksson, L., Johansson, E., y Wikstrom, C., (1998). Mixture design-design generation, PLS analysis, and model usage. Chemometrics and Intelligent Laboratory Systems, 43, 1-24.

Gutiérrez Pulido, H. y De La Vara Salazar, R. (2008). Análisis y Diseño de Experimentos, México D.F., México: Mc Graw-Hill Interamericana, 2-6; 480-493.

Instituto Argentino de Normalización, (2002). Norma IRAM 11601: Aislamiento térmico de edificios. Métodos de cálculo. Propiedades térmicas de los componentes y elementos de construcción en régimen estacionario.

Jiao, D., Shi, C., Yuan, Q., An, X., y Liu, Y. (2018). Mixture design of concrete using simplex centroid design method. Cement and Concrete Composites, 89, 76-88.

Kreith, F., Manglik, R., y Bohn, M. (2012). Principios de transferencia de calor, México D.F., México: Cengage Learning, 9-16.

Martina, P., Aeberhard, A., Aeberhard, M., y Corace J. (2003). Fabricación de un equipo de placa caliente para determinación de la conductividad térmica de materiales. In: Comunicaciones científicas y tecnológicas 2003. Universidad Nacional del Nordeste.

Menéndez, G., Bonavetti, V.L., y Irassar, E.F., (2008). Los Diseños de Experimentos y la Tecnología del Hormigón. Revista de la Construcción, 7 (1), 94-104.

Muñoz, A., Chejne, F., Espinel S. J., y Londoño G.C. (2006). Evaluación de la celulosa de papel y de las cenizas de carbón, como materiales aislantes alternativos, Dyna, 73 (148), $1-8$.

Neithalath, N., Weiss, J., y Olek, J. (2004). Acoustic performance and damping behavior of cellulose- cement composites. Cement and Concrete Composites, 26, 359-370.

Polo, M. (2015), "Aplicación del diseño de experimentos al estudio de nuevos bloques de construcción basados en residuos celulósicos”, Tesis de maestría (MEng), Universidad Tecnológica Nacional Facultad Regional Santa Fe, Santa Fe.

Zanuttini, M.A. (2012). Reciclado celulósico,Santa Fe, Argentina: Red Iberoamérica para la revalorización del reciclado celulósico,1-24; 33-119; 264-303. 